\title{
THE EFFECT OF TOPICALLY APPLIED HYALURONIC ACID GEL VERSUS TOPICAL CORTICOSTEROID IN THE TREATMENT OF EROSIVE ORAL LICHEN PLANUS
}

\author{
Mohamed I. Youssef ${ }^{1} B s c$, Naguiba M. El Sayed ${ }^{2} P h D$, Zeinab E. Darwis ${ }^{3} P h D$, \\ Rania A. Fahmy ${ }^{4} P h D$
}

\begin{abstract}
\end{abstract}
INTRODUCTION: Oral lichen planus is a common chronic inflammatory muco-cutaneous disease. Although the cause of oral lichen planus is not well known, T-lymphocyte infiltrate suggests a cell mediated immunological damage to basal cell layer of the epithelium. Its suggested treatment is topical corticosteroid. A promising new treatment for oral lichen planus [OLP] is the topical application of hyaluronic acid [HA], which has shown beneficial effect on wound healing.

OBJECTIVES: to evaluate the effect of hyaluronic acid gel in the treatment of erosive oral lichen planus in comparison with topically applied corticosteroids.

MATERIALS AND METHODS: a randomized, parallel, controlled clinical study was conducted on twenty patients who were randomly divided into two groups. Group I received topical application of corticosteroid (triamcinolone acetonide) 4-5 daily, for 28 days and Group II was similarly treated using hyaluronic acid $0.2 \%$ gel. Subjective symptom and Visual Analogue Scale (VAS) were recorded at base line, after 10 days and at 3 months. The objective sign clinical score was recorded at baseline, 1 and 3 months post -treatment.

RESULTS: Both groups showed decrease in the lesion size and symptoms of OLP. The HA treated group showed a significantly higher decrease in the pain score in comparison to the corticosteroid treated group. Regarding the healing score, the corticosteroid group showed superior results than that shown with the HA.

CONCLUSIONS: HA $0.2 \%$ gel is effective in the treatment of OLP, it resulted in a decrease in symptoms and signs of the disease. HA resulted in more control of pain when compared to topical corticosteroid.

KEYWORDS: Hyaluronic acid gel, topical corticosteroid, oral lichen planus.

1-Faculty of Dentistry, Alexandria University

2-Professor of Oral Medicine, Periodontology, Oral Diagnosis and Radiology, Faculty of Dentistry Alexandria University

3-Professor of Oral Pathology, Faculty of Dentistry Alexandria University

4-Lecturer of Oral Medicine, Periodontology, Oral Diagnosis and Radiology, Faculty of Dentistry, Alexandria University

Corresponding author:

E-mail: joejoe1466@gmail.com

\section{INTRODUCTION}

Lichen Planus [LP] is a common mucocutaneous chronic inflammatory condition affecting stratified squamous epithelia frequently involving primarily or exclusively the oral cavity. The oral form of LP appears more frequently than the cutaneous type; it may persist up to more than 20 years without spontaneous remission (1).

The etiology of the OLP remains vague and ambiguous, but a number of facts point out that the condition is most likely caused by immunological progression initiated by an antigen that changes the basal keratinocytes of the oral mucosa making them susceptible to cell immune response (2).
Assessment of efficiency in OLP treatment strategy is complicated by various factors: patient's psychological profile and medical/ pharmacological history [e.g. side effects, interactions] as well as compliance to maintain a good oral health. This latter aspect becomes essential if OLP is solely or also localized on the gingival surfaces [i.e.

desquamative gingivitis] and, therefore, vulnerable to common plaque-related gingivitis too (3).

The principal aims of current OLP therapy are the resolution of painful symptoms, oral mucosal erosions and ulceration, the reduction of the risk of oral cancer, and the maintenance of good oral hygiene (4).

Different therapies are described for OLP including drug therapy and surgery. In general, surgery is reserved to remove high-risk dysplastic areas. Use of drug therapy is the most 
common method for treatment of OLP. Various drugs have been used in the form of topical and systemic application for the treatment of OLP. Drugs used in topical form are corticosteroid, immunosuppressives, retinoids, and immunomodulators. Drugs which are used systemically are thalidomide, metronidazole, hydroxychloroquine, some retinoids and corticosteroids (5).

Glucocorticosteroids have been widely used due to their anti-inflammatory and immunosuppressive effects. The antiinflammatory effect includes the stimulation of the release of anti-inflammatory molecules such as lipocortin-1, lL-10 and nuclear factor-kappa B [NF-kB]. The immunosuppressant effect, however, is derived mainly from the suppression of antigen-driven $\mathrm{T}$-cell proliferation through the inhibition of IL-1 release from monocytes (6).

A promising treatment for OLP is the topical application of hyaluronic acids [HA]. In 2009 Nolan et al (7) studied the efficacy of topical hyaluronic acid where significant reduction in the symptoms have been recorded.

Hyaluronic acids [HA] are polysaccharides that occur naturally in the human body throughout connective, epithelial, and neural tissues. HA also provides two very important functions in wound healing as part of cell proliferation and migration. First, HA provides a temporary structure in the early stages of the wound. This structure helps facilitate the diffusion of nutritional supplies and helps

the wound to get rid of waste products from cell metabolism. Second, and most importantly, HA is closely involved in keratinocyte (cell type of the epidermis or outermost layer of the skin) proliferation and migration (8). Ultimately, this temporary structure is replaced, as the wound matures, by the addition of protein moleculesproteoglycans (whose function is to provide hydration and swelling pressure to the tissue enabling it to withstand compression forces) and collagen (8). Further, because HA is a hygroscopic macro-molecule, it is highly osmotic, allowing for control of hydration during periods of wound repair and the inflammatory process associated with it (when HA levels are elevated). The presence of elevated HA levels during this process is also of particular relevance to cell proliferation and migration. Due in part to HA's presence, cell anchorage to the extracellular matrix is weakened, permitting detachment and facilitating cell migration and division (9).

Hyaluronic acids [HA] is commercially available as sodium hyaluronate combined with polyvinylpyrrolidone (PVP) and glycyrrhetinic acid (10). Sodium hyaluronate coats the oral mucosa, thus enhances tissue hydration and accelerates healing. PVP is a hydrophilic polymer with mucoadherent and film-forming properties, which also enhances tissue hydration. Glycyrrhetinic acid is a breakdown product of glycyrrhizin, the active component of licorice, which has anti-inflammatory properties aid in ulcer healing. It is also used as a flavoring agent.

As a therapeutic agent, hyaluronic acid is used in numerous applications, among which are eye surgery, tissue reconstruction, degenerative and inflammatory joint diseases, synovial fluid replacement, release of chemical agents in surgical implants, encapsulation systems and controlled release of drugs and topical cosmetics. In local treatment of wounds, it is used in the form of cream, gel, or impregnated gauze to promote healing (11).

However, to the best of our knowledge very few studies have evaluated the use of HA in the management of OLP. In this present study, a comparison of the effectiveness of topically applied hyaluronic acid gel in the treatment of erosive OLP with respect to topically applied corticosteroids was performed.

\section{MATERIALS AND METHODS MATERIALS}

Materials which were used in this study are:

- Hyaluronic acid gel $0.2 \%$, supplied in the form of sodium hyaluronate. (Gengigel, Milano, EU.)

- Topical corticosteroids supplied as Triamcinolone Acetonide ointment 5 gm. (Kenacort-A orabase Pomad, Deva Holding A.S., Istanbul, Turkey)

PATIENT SELECTION

This study was performed after the approval of research ethics committee, Faculty of Dentistry, Alexandria University, and an informed consent form was signed by each patient after discussion of the treatment plan. This randomized parallel controlled clinical study was conducted on twenty patients with clinical and histological diagnosis of erosive oral lichen planus. Twenty patients (regardless of the sex) with clinical and histological diagnosis of erosive oral lichen planus were selected from the department of Oral Medicine, Periodontology, Oral Diagnosis, and Oral Radiology in Faculty of Dentistry, Alexandria University.

\section{Inclusion Criteria:}

1. Oral erosive lesions according to Andreasen classification (12).

2. Histological confirmation of oral erosive lichen planus according to the WHO clinico-pathological diagnostic criteria for lichen planus (13).

3. Willingness and ability to complete the present clinical trial.

4. Patients above 35 years of age.

\section{Exclusion Criteria:}

1. Histological signs of dysplasia.

2. Drugs associated with lichenoid reactions, or amalgam fillings.

3. Pregnant and lactating patients.

4. Patients with systemic diseases such as immunodysfunction, hematological and hepatological patients.

5. Smokers.

6. Patients manifesting extra-oral lesions.

7. Patients taking medications for LP for the last 3 months prior to therapy. 


\section{METHODS}

An informed consent form was obtained for each patient after providing detailed information and description of the study to all patients.

Diagnosis of erosive OLP was based on:

- History: including documented patient data [personal data, history, signs and symptoms, onset and duration of the disease]

- Clinical examination: including intraoral and extraoral examination [site and size of the lesions].

- Histological examination: An incisional biopsy was obtained from the most represented area of lesion under local anesthesia using blade number 15 . Tissues were then fixed in $10 \%$ neutral buffered formalin [pH 7.4] for at least 24 hours, then sent for staining and histological examination.

After clinical and histological examination, twenty patients were selected for this clinical study. All patients were subjected to oral hygiene instructions and removal of all source of traumatic irritation. They were randomly assigned into 2 groups:

- Group I: Ten erosive OLP patients who were treated by topical corticosteroids in orabase 4-5 times daily for 28 days (14).

- Group II: Ten erosive OLP patients who were treated by hyaluronic acid gel 4-5 times daily for 28 days (15).

\section{Clinical evaluation:}

Oral lesions were evaluated before and after therapy, the response to this treatment was measured according to reduction in signs and symptoms [pain] scores using:

\section{Subjective evaluation:}

Visual Analog Scale [VAS] is a simple measurement tool that tries to measure the intensity of certain sensations and feelings of pain of the patient.it ranges across a continuum from none to an extreme amount of pain in straight horizontal $10 \mathrm{~cm}$ line [from 0-100 mm] (16).

At the beginning of the study, patients were asked to grade overall severity of their symptoms on the VAS then it was reassessed after 10 days and at the end of the 3 month period using same scale. The difference between these scores in $\mathrm{mm}$ was expressed as percentages to document any improvement from patient's view, the overall response was categorized compared with baseline as following: (17)

- Poor: $25 \%$

- Fair: 25 - $50 \%$

- Good: 50 - $75 \%$

- Excellent: more than $75 \%$

\section{Objective evaluation:}

The lesion was evaluated and examined into clinical scores, according to Thongprasom et al. (18). It is an assignment of one of the following scores in atrophic/erosive OLP:

- Score 0: no lesion.

- Score 1: mild white striae.

- Score 2: white striae with erythematous area less than 1 $\mathrm{cm} 2$.
- Score 3: white striae with erythematous area more than 1 cm2.

- Score 4: white striae with erosive area less than $1 \mathrm{~cm} 2$.

- Score 5: white striae with erosive area more than $1 \mathrm{~cm} 2$.

Objective evaluation was done at the beginning of the study [baseline], and then it was reassessed after 1 month and 3 months.

\section{Statistical analysis}

Data were fed to the computer and analyzed using IBM SPSS software package version 20.0. Qualitative data were described using number and percent. Quantitative data were described using range (minimum and maximum), mean, standard deviation and median. Comparison between different groups regarding categorical variables was tested using Wilcoxon signed ranks test. For normally distributed data, comparison between more than two population were analyzed F-test (ANOVA) to be used. Kruskal Wallis test was used to compare between different groups and pair wise comparison was assessed using Mann-Whitney test, Significance of the obtained results was judged at the $5 \%$ level.

\section{RESULTS}

Twenty patients suffering from erosive OLP were enrolled in this study. They were randomly allocated into two groups [ten patients per treatment group]. Patients of group I received topical corticosteroids in orabase 4-5 times daily for 28 days, while patients of group II received hyaluronic acid gel 4-5 times daily for 28 days. All patients had visits for clinical evaluation after one and three months. Subjective and objective assessments were recorded at each visit. All patients complied to the follow up visits, no adverse reactions were recorded in any of the treated patients. No exacerbation or recurrence of lesions were recorded for any of the treated patients even after cessation of treatment.

Table [1], figure [1] are showing the pain difference between the test groups according to VAS scale. In group I the mean pain difference was $1.60 \pm 0.52$ while in group II it was $6.80 \pm 1.55$. There was a significant difference between the two study groups regarding the pain difference [p>0.05].

Table 1: Comparison between the two studied groups regarding the the pain differance

\begin{tabular}{|l|c|c|c|c||}
\hline & $\begin{array}{c}\text { Group I } \\
\text { Corticosteroids } \\
\text { [n= 10] }\end{array}$ & $\begin{array}{c}\text { Group II } \\
\text { Hyaluronic } \\
\text { acid } \\
\text { [n= 10] }\end{array}$ & $\mathbf{Z}$ & $\mathbf{p}$ \\
\hline Difference & & & & \\
Min. - Max. & $1.0-2.0$ & $4.0-8.0$ & & \\
Mean \pm SD. & $1.60 \pm 0.52$ & $6.80 \pm 1.55$ & 3.87 & $<0.001$ \\
Median & 2.0 & 7.0 & $7^{*}$ & ${ }^{*}$ \\
\hline
\end{tabular}

$\mathrm{Z}, \mathrm{p}: \mathrm{Z}$ and $\mathrm{p}$ values for Mann Whitney test.

*: Statistically significant at $\mathrm{p} \leq 0.05$ 


\section{The two studied groups regarding the pain difference percentage}

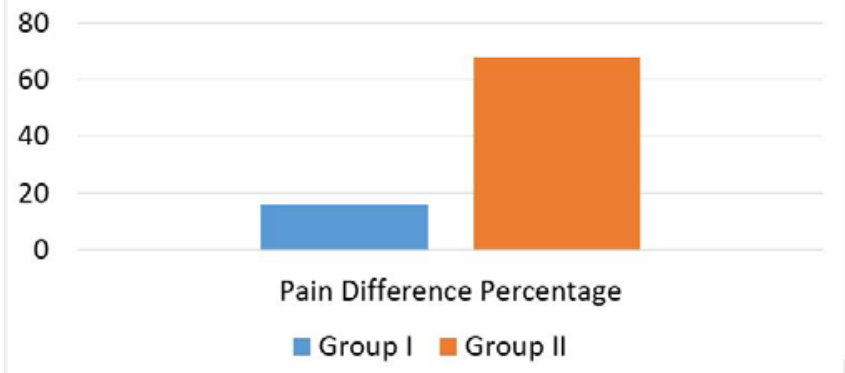

Figure 1: The two studied groups regarding the pain difference

Table [2], figure [2] are showing the results comparing the clinical healing after one month and 3 months for each group. In group I; after one month, the mean of the clinical score of healing was $2.40 \pm 0.52$ while after 3 months, the mean of the score was $1.0 \pm 0.67$.

There was a significant difference between one and three months regarding the clinical score of healing [p<0.05]. As for group II, after one month, the mean of the score was 3.20 \pm 0.79 while after 3 months, the mean was $1.80 \pm 0.42$. There was a significant difference between the one month and three months regarding this score $[\mathrm{P}<0.05]$.

Table 2: Comparison between the two studied groups according to the clinical score of healing

\begin{tabular}{||l|l|l|l|l||}
\hline Score & $\begin{array}{l}\text { Corticosteroids } \\
{[\mathbf{n}=\mathbf{1 0}]}\end{array}$ & $\begin{array}{l}\text { Hyaluronic acid } \\
{[\mathbf{n = 1 0 ]}}\end{array}$ & $\mathbf{Z}$ & $\mathbf{p}$ \\
\hline One Month & & & & \\
Min. - Max. & $2.0-3.0$ & $2.0-4.0$ & & 0.023 \\
Mean \pm SD. & $2.40 \pm 0.52$ & $3.20 \pm 0.79$ & $2.274^{*}$ & $*$ \\
Median & 2.0 & 3.0 & & \\
\hline Three Month & & & & \\
Min. - Max. & $0.0-2.0$ & $1.0-2.0$ & & \\
Mean \pm SD. & $1.0 \pm 0.67$ & $1.80 \pm 0.42$ & $2.684^{*}$ & $* .007$ \\
Median & 1.0 & 2.0 & & \\
\hline
\end{tabular}

Z, p: Z and p values for Mann Whitney test.

*: Statistically significant at $\mathrm{p} \leq 0.05$

\section{The two studied groups according to the clinical score of healing}

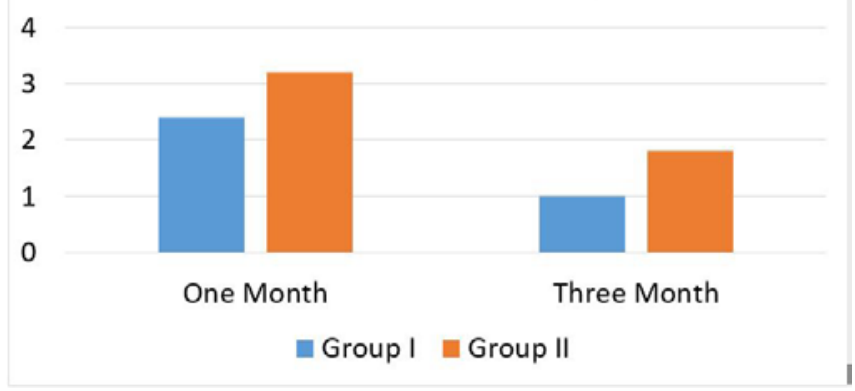

Figure 2: The two studied groups according to the lesion size

\section{DISCUSSION}

The management of OLP is very difficult due to its chronic nature that requires a long-term treatment (3). The World Health Organization [WHO] considers OLP as "a generalized state associated with a significant increased risk of cancer”. The cause of increased oral cancer risk in OLP patients is unknown; nonetheless the highest rate of transformation is found with the erosive and erythematic forms when left untreated (19).

In general, according to the European Association of Oral Medicine [EAOM] guidelines, (3) OLP treatment is necessary only when it is symptomatic. For the treatment of oral mucosal conditions, local drug delivery is a more efficient approach than the systemic administration. The reasons to prefer a local drug delivery to treat the oral soft tissue diseases are linked to their high frequency, their chronic status [requiring chronic treatment regimens] and above all to their excellent response to topical drugs.

In the current study, corticosteroids were found to have a significant effect on the healing of the lesions while on the other hand pain relief was not with the same potency. The favorable healing effect of topically applied corticosteroids have been recorded in several studies.

$\mathrm{Up}$ to the present time, the application of immunosuppressive drugs, such as glucocorticoids, appears to be the safest and most effective topical treatment available, even if there is much dispute about their long-term efficacy and benefits, and there is a wide scenario with several different remedies proposed, at least of auxiliary benefit.

In fact, the bioavailability of circulating and/or endogenous hydrocortisone [cortisol] in epidermal cells is a key determinant in inflammatory disease. However, it is unknown whether epidermal cells can regulate tissue cortisol and whether they are capable of producing endogenous glucocorticoids (3). A further key observation is that keratinocytes responded to stimulation with ACTH by a significant increase in the de novo synthesis of cortisol. The same authors have recently reported that epidermal keratinocytes can be considered a new non-adrenal glucocorticoid- system endogenously implicated in regulation of cortisol: data showed that oral fibroblasts and keratinocytes were able to activate cortisone into the active form cortisol, and confirmed the capacity of synthesizing cortisol de novo following stimulation with ACTH; particularly, when the isoenzyme11b-Hydroxysteroid dehydrogenase [HSD] $1 / 2$ is present, it controls cortisol deactivation. Supplementary close examinations to control bioavailability of circulating cortisol in epidermal cells are awaited, and well-designed randomized controlled trials should be carried out to establish the accurate effectiveness of topical administration for the treatment of immunologically mediated oral mucosal disease as OLP.

Also due to the chronic nature of OLP disease it requires long-term corticosteroids use which was reported to have adverse effects (10). It should be underlined that prolonged use of topical steroids may lead to the development of 
candidiasis. That is why candidal cultures are important as routine investigation, before, during or after treatment. Also, other rare adverse effects were observed, such as: bad taste and smell, dry mouth, swollen mouth, nausea (9). Hence, a safer and more effective therapy for symptomatic OLP is necessary $(20,21)$.

The potential for adrenal suppression with prolonged use have also been implicated, which necessitates careful and frequent follow-up. Adrenal suppression seems to be more common when steroids are used as mouthwashes (6).

A promising new treatment for OLP is the topical form application of $0.2 \%$ hyaluronic acid. HA $0.2 \%$ gel offers advantages over topical steroids in that it is safe to be used in all grades of oral ulceration (10) and the only contraindication known is the presence of history of allergy or hypersensitivity to HA or any of the ingredients.

The synovial fluid, umbilical cord, skin and rooster comb, or from bacteria through a process of fermentation or direct isolation are the sources of commercially produced HA. Studies on the properties of HA like chemical and physicochemical and on its physiological role in humans have been done extensively (22). HA is an ideal biomaterial for cosmetic, medical and pharmaceutical applications due to its versatile properties such as its biocompatibility, nonimmunogenicity, biodegradability and viscoelasticity. A variety of mechanisms of the tissue healing properties of HA have been identified (23).

Signifying the healing effects of HA the present study demonstrated reduction in the degree of pain and erythema for the treated patients. There was a significant reduction of pain in patients treated with HA when compared to the corticosteroid group. This indicate that the patients' satisfaction and tolerance to the HA treatment was more than that to the corticosteroid treatment. However, corticosteroid treated patients showed significantly higher healing effect than those treated with HA.

In accordance to the present results, Nolan et al reported a significant reduction in soreness/pain scores using the VAS scores in HA treated OLP patients when compared with placebo. However, both placebo and HA had no effect on the extent and severity of the OLP as described by Thongprasom's criteria for clinical healing score. Similarly in 2016 Shetty et al (24) have reported reduction in VAS scores and a significant improvement in relief of symptoms in the HA treated OLP patients as compared to placebo. Statistically significant improvements were observed in the objective criteria which involved the degree of erythema and the mean area of the lesions with HA application when compared to the control group on placebo.

The previous had compared the effect of HA to a placebo. To the best of our knowledge no studies have compared the effect of HA to topically applied corticosteroid in the management of OLP. A major issue is whether a placebo randomized controlled clinical trial [RCT] is ethically acceptable for symptomatic patients with OLP. In fact, on one hand, placebo-controlled trials of local steroids are missing, and on the other hand, local steroids are universally recognized as first line treatment, and for this reason perhaps steroids [and not placebo] should be the ideal control group (25).

Although the effect of HA regarding the healing of OLP was not superior to that reported by the topically applied corticosteroid in the current study, several studies have reported the beneficial effect of HA in healing of different lesions. In a systematic review, studies on topical formulations containing $\mathrm{HA}$ evaluated the treatment of chronic wounds of various etiologies, such as thermal injury, venous ulcers and diabetic foot concluding that the product promotes healing when compared to other treatments (26).

In a RCT, the efficacy of hyaluronic acid in the treatment of venous ulcers was compared to a neutral carrier. The primary outcome was the percentage reduction of the wound size at 45 days. It was found that the reduction of the ulcer surface area was significantly higher in the intervention group $(73 \pm 4.6 \%)$ versus the control group $(46 \pm 9.6 \%)(\mathrm{P}=$ $0.011)$. The number of healed ulcers in the intervention group was higher at day 45 (31\% vs. 9\%, respectively) and day 60 (37\% vs. 16\%, respectively) ( $\mathrm{P}<0.05)(11)$.

Specifically in thermal injuries, a cohort study with 60 patients with partial thickness burns found an average reduction of $50 \%$ in the size of the wound in the first five follow up days of combined application of hyaluronic acid with zinc. Complete healing occurred in 93.3\% of the sample after 21 days of follow up, with an average healing time of 10.5 days (27).

In experimental studies, HA demonstrated superiority compared to other covers, with respect to shorter time of wound healing and histological characteristics such as improved elasticity and higher microvascular density (28, 29).

The beneficial effect of hyaluronic acid in wounds healing may be attributed to the improved water retention, which favors a suitable environment for the formation of collagen and elastin, and allows the cells to proliferate and differentiate, accelerating the healing process (30). Furthermore, the anti-inflammatory properties of hyaluronic acid influence the healing, preventing the conversion of wound $(31,32)$ and formation of hypertrophic scars or keloids (33).

Another relevant aspect for the application of HA is the fact that this biomaterial is a non-immunogenic substance. An experimental study was conducted to determine the skin tolerance to topical application of hyaluronic acid in full thickness injuries (35\% of the body surface area), and it showed the substance was well tolerated and there were no adverse or side effects (34).

Therefore a larger sample of patients with longer follow up periods are required to further evaluate and establish the long term efficacy of topical hyaluronic acid in the treatment of erosive OLP.

A combination of both topically applied hyaluronic acid and corticosteroids is suggested to be used in the treatment of 
erosive OLP to gain the best out of both treatments for patients' benefit.

\section{CONCLUSIONS}

Based on the results of the present study it was concluded that the topical application of hyaluronic acid $0.2 \%$ appears to be significantly more effective in the control of the symptoms of OLP when compared to topically applied Triamcinolone Acetonide ointment. Topical hyaluronic acid appears to be well tolerated when used on the oral mucous membrane in all the patients. It also proved to be effective in controlling but not eradicating OLP lesions in some patients.

\section{CONFLICT OF INTEREST}

The authors declare that they have no conflicts of interest.

\section{REFERENCES}

1. Bajaj DR, Khoso NA, Devrajani BR, Matlani BL, Lohana P. Oral lichen planus: a clinical study. J Coll Physicians Surg Pak. 2010;20:154-7.

2. Le Cleach L, Chosidow O. Clinical practice. Lichen planus. N Engl J Med. 2012;366:723-32.

3. Bagan J, Compilato D, Paderni C, Campisi G, Panzarella $\mathrm{V}$, Picciotti $\mathrm{M}$, et al. Topical therapies for oral lichen planus management and their efficacy: a narrative review. Curr Pharm Des. 2012;18:5470-80.

4. Sugerman P, Sabage N. Oral lichen planus: causes, diagnosis and management. Australian dental journal. 2002;47:290-7.

5. Oliveira Alves MG, Almeida JD, Balducci I, Guimarães Cabral LA. Oral lichen planus: A retrospective study of 110 Brazilian patients. BMC Research Notes. 2010;3:1-4.

6. Gonzalez-Moles MA, Scully C. Vesiculo-erosive oral mucosal disease--management with topical corticosteroids: (2) Protocols, monitoring of effects and adverse reactions, and the future. J Dent Res. 2005;84:3028.

7. Nolan A, Badminton J, Maguire J, Seymour RA. The efficacy of topical hyaluronic acid in the management of oral lichen planus. J Oral Pathol Med. 2009;38:299-303.

8. Bourguignon LY, Ramez M, Gilad E, Singleton PA, Man MQ, Crumrine DA, et al. Hyaluronan-CD44 interaction stimulates keratinocyte differentiation, lamellar body formation/secretion, and permeability barrier homeostasis. J Invest Dermatol. 2006;126:1356-65.

9. Culp LA, Murray BA, Rollins BJ. Fibronectin and proteoglycans as determinants of cell-substratum adhesion. Journal of supramolecular structure. 1979;11:401-27.

10.Smith T. Gelclair: managing the symptoms of oral mucositis. Hosp Med. 2001;62:623-6.

11. Humbert P, Mikosinki J, Benchikhi H, Allaert FA. Efficacy and safety of a gauze pad containing hyaluronic acid in treatment of leg ulcers of venous or mixed origin: a double-blind, randomised, controlled trial. Int Wound J. 2013;10:159-66.
12. Liguori V, Guillemin C, Pesce GF, Mirimanoff RO, Bernier J. Double-blind, randomized clinical study comparing hyaluronic acid cream to placebo in patients treated with radiotherapy. Radiother Oncol. 1997;42:15561.

13. Rad M, Hashemipoor MA, Mojtahedi A, Zarei MR, Chamani G, Kakoei S, et al. Correlation between clinical and histopathologic diagnoses of oral lichen planus based on modified WHO diagnostic criteria. Oral Surg Oral Med Oral Pathol Oral Radiol Endod. 2009;107:796-800.

14. Edwards PC, Kelsch R. Oral lichen planus: clinical presentation and management. J Can Dent Assoc. 2002;68:494-9.

15.Aghahosseini F, Arbabi-Kalati F, Fashtami LA, Djavid GE, Fateh M, Beitollahi JM. Methylene blue-mediated photodynamic therapy: a possible alternative treatment for oral lichen planus. Lasers Surg Med. 2006;38:33-8.

16. Huskisson EC, Jones J, Scott PJ. Application of visualanalogue scales to the measurement of functional capacity. Rheumatol Rehabil. 1976;15:185-7.

17.Passeron T, Zakaria W, Ostovari N, Mantoux F, Lacour JP, Ortonne JP. Treatment of erosive oral lichen planus by the $308 \mathrm{~nm}$ excimer laser. Lasers Surg Med. 2004;34:205.

18. Canto AM, Muller H, Freitas RR, Santos PS. Oral lichen planus (OLP): clinical and complementary diagnosis. An Bras Dermatol. 2010;85:669-75.

19. Thongprasom K, Carrozzo M, Furness S, Lodi G. Interventions for treating oral lichen planus. Cochrane Database Syst Rev. 2011:CD001168.

20. Hegarty AM, Hodgson TA, Lewsey JD, Porter SR. Fluticasone propionate spray and betamethasone sodium phosphate mouthrinse: a randomized crossover study for the treatment of symptomatic oral lichen planus. J Am Acad Dermatol. 2002;47:271-9.

21. Vente C, Reich K, Rupprecht R, Neumann C. Erosive mucosal lichen planus: response to topical treatment with tacrolimus. Br J Dermatol. 1999;140:338-42.

22. Brown MB, Jones SA, Weijiang H, Martin GP, editors. Hyaluronan: Investigations into the mode of action of hyaluronan in topical drug delivery. ACS symposium series; 2006: Oxford University Press.

23. David-Raoudi M, Tranchepain F, Deschrevel B, Vincent JC, Bogdanowicz P, Boumediene K, et al. Differential effects of hyaluronan and its fragments on fibroblasts: relation to wound healing. Wound Repair Regen. 2008;16:274-87.

24. Shetty RR, Burde KN, Guttal KS. The Efficacy of Topical Hyaluronic Acid $0.2 \%$ in the Management of Symptomatic Oral Lichen Planus. J Clin Diagn Res. 2016;10:ZC46-50.

25. Normile D. Ethics. Clinical trials guidelines at odds with U.S. policy. Science. 2008;322:516.

26. Voigt J, Driver VR. Hyaluronic acid derivatives and their healing effect on burns, epithelial surgical wounds, and chronic wounds: A systematic review and meta-analysis 
of randomized controlled trials. Wound Repair and Regeneration. 2012;20:317-31.

27. Juhasz I, Zoltan P, Erdei I. Treatment of partial thickness burns with Zn-hyaluronan: lessons of a clinical pilot study. Ann Burns Fire Disasters. 2012;25:82-5.

28. Shimizu N, Ishida D, Yamamoto A, Kuroyanagi M, Kuroyanagi Y. Development of a functional wound dressing composed of hyaluronic acid spongy sheet containing bioactive components: evaluation of wound healing potential in animal tests. J Biomater Sci Polym Ed. 2014;25:1278-91.

29. Yang G, Espandar L, Mamalis N, Prestwich GD. A crosslinked hyaluronan gel accelerates healing of corneal epithelial abrasion and alkali burn injuries in rabbits. Vet Ophthalmol. 2010;13:144-50.

30. Anilkumar T, Muhamed J, Jose A, Jyothi A, Mohanan P, Krishnan LK. Advantages of hyaluronic acid as a component of fibrin sheet for care of acute wound. Biologicals. 2011;39:81-8.

31. Neuman MG, Nanau RM, Oruna-Sanchez L, Coto G. Hyaluronic acid and wound healing. J Pharm Pharm Sci. 2015;18:53-60.

32. Sun LT, Friedrich E, Heuslein JL, Pferdehirt RE, Dangelo NM, Natesan S, et al. Reduction of burn progression with topical delivery of (antitumor necrosis factor-alpha)hyaluronic acid conjugates. Wound Repair Regen. 2012;20:563-72.

33. Hoffmann A, Hoing JL, Newman M, Simman R. Role of Hyaluronic Acid Treatment in the Prevention of Keloid Scarring. J Am Coll Clin Wound Spec. 2012;4:23-31.

34. Weinstein-Oppenheimer CR, Aceituno AR, Brown DI, Acevedo C, Ceriani R, Fuentes MA, et al. The effect of an autologous cellular gel-matrix integrated implant system on wound healing. J Transl Med. 2010;8:59. 